\title{
Ion fluxes through nanopores and transmembrane channels
}

\author{
J. R. Bordin, ${ }^{1}$ A. Diehl, ${ }^{2}$ M. C. Barbosa,${ }^{3}$ and Y. Levin ${ }^{3}$ \\ ${ }^{1}$ Programa de Pós-Graduação em Física, Instituto de Física, Universidade Federal do Rio Grande do Sul Caixa Postal 15051, \\ CEP 91501-970, Porto Alegre, RS, Brazil \\ ${ }^{2}$ Departamento de Física, Instituto de Física e Matemática,Universidade Federal de Pelotas, Caixa Postal 354, \\ CEP 96010-900, Pelotas, RS, Brazil \\ ${ }^{3}$ Instituto de Física, Universidade Federal do Rio Grande do Sul Caixa Postal 15051, CEP 91501-970, Porto Alegre, RS, Brazil
}

(Received 26 August 2011; revised manuscript received 8 February 2012; published 26 March 2012)

\begin{abstract}
We introduce an implicit solvent Molecular Dynamics approach for calculating ionic fluxes through narrow nanopores and transmembrane channels. The method relies on a dual-control-volume grand-canonical molecular dynamics (DCV-GCMD) simulation and the analytical solution for the electrostatic potential inside a cylindrical nanopore recently obtained by Levin [Europhys. Lett. 76, 163 (2006)]. The theory is used to calculate the ionic fluxes through an artificial transmembrane channel which mimics the antibacterial gramicidin A channel. Both current-voltage and current-concentration relations are calculated under various experimental conditions. We show that our results are comparable to the characteristics associated to the gramicidin A pore, especially the existence of two binding sites inside the pore and the observed saturation in the current-concentration profiles.
\end{abstract}

DOI: 10.1103/PhysRevE.85.031914

PACS number(s): 87.16.A-, 87.10.Tf, 87.16.Uv

\section{INTRODUCTION}

Ion channels are structures formed when specific proteins are incorporated into the phospholipid membrane [1]. The channels serve to establish an electrostatic potential gradient across the cell membrane by allowing an ion-specific flux to pass through the membrane. There are many different ion channels in living cells. They differ in composition, pore structure, and ion selectivity [2,3]. Thus, a full description of the architecture and operation of ion channels is a very difficult task. From purely electrostatic point of view, operation of ion channels presents an interesting theoretical puzzle. Since the channel passes through a low-dielectric membrane, there exists a large potential energy barrier for ionic solvation inside the pore. Yet, in practice, it is well known that when open, ion channels sustain a very large ionic transport rate, compatible with a free diffusion.

A recent advance in nanoscale technology is the synthesizing of nanotubes with chemical modified surface [4], which allows one to construct functionalized nanotubes that mimic the behavior of biological ion channels and are stable when inserted in a lipid bilayer [5,6], and also exhibit a high-energy barrier [7]. An excellent review of recent theoretical advances in synthetic nanotubes which mimic the behavior of biological ion channels is given by Hilder et al. [8].

There have been a number of different strategies proposed to understand the ion transport across biological and synthetic channels. One approach used extensively over the last few years is all-atom molecular dynamics (MD) simulation. The advantage of atomistic MD is that molecular structures of the pore, ionic species, and water are taken explicitly into account [3,7-14]. Although this method is very appealing, it remains a huge task to relate the measured observables to the experimental results. One of the main obstacles is the computational times needed to achieve the time scales observed experimentally [15-18], which, in most cases, require large-scale MD simulations in special-purpose machines [19]. Another problem is the choice of the molecular force field to be used. For instance, in the case of the biological channel gramicidin A (gA), one of the most studied ionic channels, the first MD simulations have predicted a potential of mean force (PMF) with a barrier much larger than expected experimentally [17]. Recent MD simulations with a molecular force field properly constructed appear to produce PMFs in semiquantitative agreement with experiments [20-22]. Although promising, this agreement seems to be very sensitive to the molecular force field used and with the membrane where the gA channel is embedded [22]. Furthermore, the classical water models used in all-atom simulations are parametrized for bulk water and might show erroneous behavior in a strongly confined environment. Such artifacts of classical water and ion models have been recently observed in the studies of ionic solvation near interfaces, when compared to the full $a b$ initio simulations [23]. It has been shown that for ionic solvation in an interfacial geometry properly constructed dielectric continuum models agree better with results of full $a b$ initio simulations than the classical explicit water models [23-25].

One alternative to atomistic MDs are the, so-called, Brownian dynamics (BD) simulation [10,26,27]. In these simulations only ionic movement is integrated, while the protein degrees of freedom are held fixed and the water is treated as a uniform dielectric continuum. This significantly reduces the computational cost of the simulation, allowing one to access much larger time scale. Nevertheless, BD simulations still require solution of Poisson partial-differential equation at each new time step of the simulation, making them quite difficult to implement. Recently new atomic-resolution BD simulations have been proposed [28]. Using PMFs derived from allatom MD simulations, this method was able to access large simulation times at reasonably low computational cost [28].

Thus, in order to avoid the difficulties of all-atom and BD simulations, as well as to be able to explore large time scales necessary for measuring the transmembrane currents, it might be useful to use the dielectric continuum models of ion channels as a first-order approximation for the transmembrane dynamics. In this paper we explore the transmembrane ion fluxes using the continuum electrostatics model recently 
introduced by Levin [29]. Briefly stated, Levin solved the Poisson equation with the appropriate boundary conditions to obtain an analytical expression for the electrostatic interaction potential (Green function) between the charges inside a finite cylindrical pore passing through a low-dielectric phosphoric membrane. This electrostatic potential can be used to calculate the forces acting between the ions inside the pore and between the ions and the charged protein residues embedded in the cell membrane. It is our goal in this study to use the interaction potential derived by Levin in a series of Dual-Control-Volume Grand-Canonical Molecular Dynamics (DCV-GCMD) [30,31] simulations of a simple model of narrow transmembrane channel. We should stress that our model is very different from the mean-field Poisson-Nernst-Plank (PNP) theory [32,33], where the Poisson equation and the continuity equations for mobile ions are solved simultaneously in a self-consistent way. For narrow channel such as gA the correlation effects between the ions are of fundamental importance and the mean-field description of ionic conduction is bound to fail [34].

Our simulations are performed for different electrolyte concentrations and external applied electric fields to verify if our model reproduces the ionic flow obtained for one specific system: the gA channel. The comparison between our model and experiments for various concentrations and external fields is an indication that it is able to reproduce the observed ionic fluxes in narrow channels [8,35].

The paper is organized as follows. The model and computational details are given in Sec. II. The results are discussed in Sec. III, and the summary and conclusions are presented in Sec. IV.

\section{THE MODEL SYSTEM AND THE SIMULATION METHODOLOGY}

\section{A. A model for transmembrane channels}

We use the channel-reservoir setup illustrated schematically in Fig. 1 to calculate the ionic currents due to electrostatic potential gradients. The simulation box, a cubic parallelepiped with volume $5 L \times L \times L$ and $L=20 \AA$, contains two reservoirs (two control volumes $\mathrm{CV}_{1}$ and $\mathrm{CV}_{2}$, within which the chemical potentials of the ionic species are maintained constant) and a membrane with a channel connecting these two reservoirs through small buffer regions. The channel structure, a simplified model of a transmembrane pore, is built as a cylindrical tube, with radius $a=3 \AA$ and length $L_{c}=35 \AA$, made of stationary Lennard-Jones (LJ) spheres of diameter $\sigma_{c}=2 \AA$.

Both sides of the channel structure, except for the orifices, are bound by confining walls, as well as the two extremes of the simulation box in the $x$ direction; see Fig. 1 . The system contains positive and negative ions with diameters $\sigma_{+}$and $\sigma_{-}$, respectively, inside a structureless solvent of dielectric constant $\epsilon_{w}=80$, and the same value of dielectric is used inside and outside the channel, while the membrane has a dielectric constant equal to $\epsilon_{m}=2$, both in units of vacuum permittivity. It should be noted that in confined environments the dielectric constant of water could be considerably lower than the bulk value $80[36,37]$. However, in this work we follow the same prescription of previous dielectric continuum models, where the use of same dielectric constant is compensated by fixing a lower diffusion constant for the positive ions moving inside the channel [10,38].

The particle-particle interactions are separated into shortand long-range contributions, while the particle-channel has only a short-range interaction. For the short-range part we will use the WCA LJ potential [39]:

$$
U_{i j}^{\mathrm{WCA}}(r)= \begin{cases}U_{\mathrm{LJ}}(r)-U_{\mathrm{LJ}}\left(r_{c}\right), & r \leqslant r_{c}, \\ 0, & r>r_{c},\end{cases}
$$

where $U_{\mathrm{LJ}}(r)$ is the standard 12-6 LJ potential. The cutoff distance is $r_{c}=2^{1 / 6} \sigma_{i j}$, where $\sigma_{i j}=\left(\sigma_{i}+\sigma_{j}\right) / 2$ is the centerto-center separation between an ion of species $i$ (cation or anion) and a particle of species $j$ (cation, anion, or fixed LJ channel sphere) separated by a distance $r$. The confining walls in simulation box extremes and surrounding the channel structure are modeled with the same WCA LJ potential, however considering the $x$-projection of the distance between one ion in the bulk and the wall position. The long-range contribution is calculated depending on the region where the ion is located.

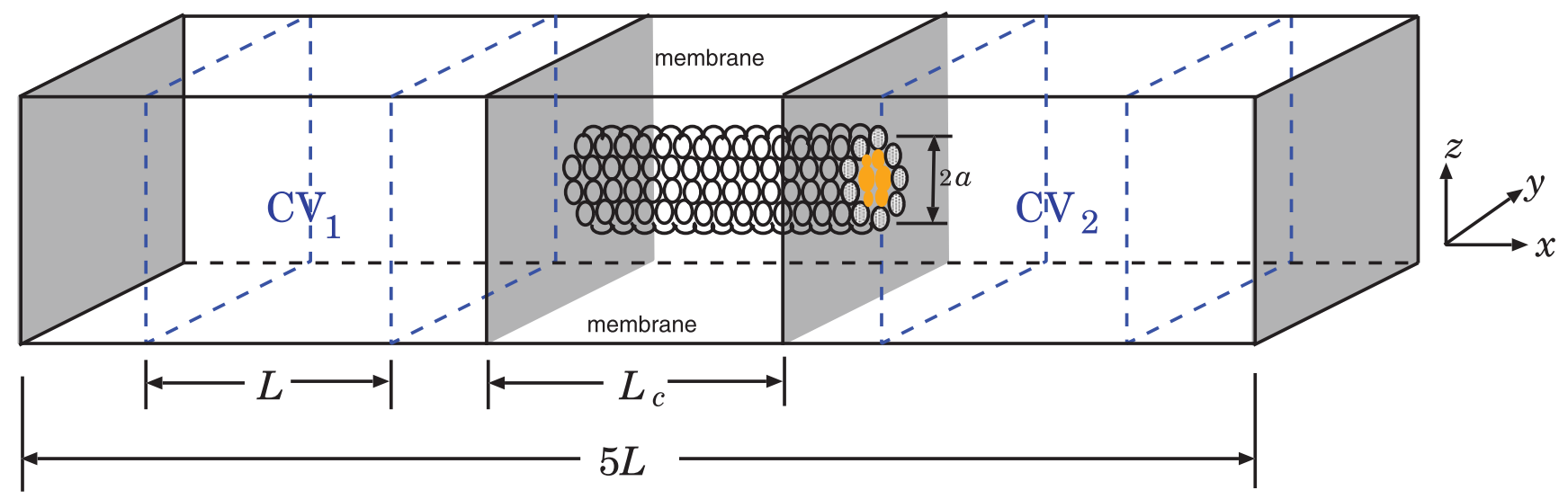

FIG. 1. (Color online) Schematic depiction of the simulation cell. The ionic channel is built as a cylinder made of a sequence of annular rings of LJ spheres. The system is confined in $x$ direction by two flat walls (represented by gray) at each end of the simulation box. The same flat walls are used to maintain the flux of cations between the two reservoirs (CVs) through the channel. Periodic boundary conditions are applied in both $y$ and $z$ directions. 
For the regions outside the channel, the interaction energy between the two ions is the usual Coulomb potential

$$
U_{i j}^{\text {coul }}(r)=\frac{1}{4 \pi \epsilon_{w}} \frac{q_{i} q_{j}}{r_{i j}}
$$

where $r_{i j}$ is the distance between the two ions. The infinite extent of the particle reservoir is taken into account using the Ewald summation.

For the region inside the channel we will use the model introduced by Levin [29]. In this model ions inside the pore interact through the electrostatic potential which is only a function of their separation in the $x$ direction (along the axis of symmetry of the channel). This is quite reasonable for narrow channels that allows only single-file motion. When one ion with charge $q$ enters into the channel it interacts with the other ions and with the $p$ residues of charge $-q$ embedded into the channel wall at transverse distance $\rho$ from the central axis. In addition to the electrostatic interaction between the ions and the residues, there is a self-energy penalty $U$ associated with an ion entering into the region surrounded by the low-dielectric material. The electrostatic energy of the ions inside the pore is then

$$
\begin{aligned}
V= & \frac{1}{2} \sum_{i=1}^{N} \sum_{j=1}^{N} q_{i} \varphi_{\text {in }}\left(x_{i}, x_{j}\right)+\sum_{i=1}^{N} \sum_{j=1}^{p} q_{i} \varphi_{\text {out }}\left(x_{i}, \rho, x_{j}\right) \\
& +\sum_{i=1}^{N} q_{i} U\left(x_{i}\right),
\end{aligned}
$$

where $\quad \varphi_{\text {in }}\left(x_{i}, x_{j}\right)=\varphi_{1}\left(x_{i}, x_{j}\right)+\varphi_{2}\left(x_{i}, x_{j}\right) . \quad$ The $\quad$ two electrostatic potentials are [29]

$$
\varphi_{1}\left(x_{i}, x_{j}\right)=\frac{q}{\epsilon_{w}} \int_{0}^{\infty} d k \frac{\left\{\alpha^{2}(k) e^{k\left|x_{i}-x_{j}\right|-2 k L_{C}}+\alpha(k) \beta(k)\left[e^{-k\left(x_{i}+x_{j}\right)}+e^{\left.k\left(x_{i}+x_{j}\right)-2 k L_{c}\right)}\right]+\beta^{2}(k) e^{-k\left|x_{i}-x_{j}\right|}\right\}}{\beta^{2}(k)-\alpha^{2}(k) \exp \left[-2 k L_{C}\right]},
$$

where $\alpha=\left[k-\left(k^{2}+\kappa^{2}\right)^{1 / 2}\right]$ and $\beta=\left[k+\left(k^{2}+\kappa^{2}\right)^{1 / 2}\right]$, with $\kappa$ the inverse Debye length that characterizes the electrolyte concentration in the two reservoirs, and

$$
\varphi_{2}\left(x_{i}, x_{j}\right)=\frac{4 q\left(\epsilon_{w}-\epsilon_{p}\right)}{\epsilon_{w} L_{c}} \sum_{n=1}^{\infty} \frac{K_{0}\left(k_{n} a\right) K_{1}\left(k_{n} a\right) \sin \left(k_{n} x_{i}\right) \sin \left(k_{n} x_{j}\right)}{\epsilon_{w} I_{1}\left(k_{n} a\right) K_{0}\left(k_{n} a\right)+\epsilon_{p} I_{0}\left(k_{n} a\right) K_{1}\left(k_{n} a\right)},
$$

where $I_{n}$ and $K_{n}$ are the modified Bessel functions of order $n$. Here $a$ is the channel radius and $k_{n}=n \pi / L_{c}$. The interaction potential between an ion and a fixed charged residue is

$$
\varphi_{\text {out }}\left(x_{i}, \rho, x_{j}\right)=\frac{4 q}{L_{c}} \sum_{n=1}^{\infty} \frac{1}{k_{n} a} \frac{K_{0}\left(k_{n} \rho\right) \sin \left(k_{n} x_{i}\right) \sin \left(k_{n} x_{j}\right)}{\epsilon_{w} I_{1}\left(k_{n} a\right) K_{0}\left(k_{n} a\right)+\epsilon_{p} I_{0}\left(k_{n} a\right) K_{1}\left(k_{n} a\right)} .
$$

Finally, the electrostatic potential responsible for the barrier is given by

$$
\begin{aligned}
U(x)= & \frac{q}{2 \epsilon_{w}} \int_{0}^{\infty} d k \frac{\left\{2 \alpha^{2}(k) e^{-2 k L_{C}}+\alpha(k) \beta(k)\left[e^{-2 k x}+e^{2 k\left(x-L_{c}\right)}\right]\right\}}{\beta^{2}(k)-\alpha^{2}(k) \exp \left[-2 k L_{C}\right]} \\
& +\frac{2 q\left(\epsilon_{w}-\epsilon_{p}\right)}{\epsilon_{w} L_{c}} \sum_{n=1}^{\infty} \frac{K_{0}\left(k_{n} a\right) K_{1}\left(k_{n} a\right) \sin ^{2}\left(k_{n} x\right)}{\epsilon_{w} I_{1}\left(k_{n} a\right) K_{0}\left(k_{n} a\right)+\epsilon_{p} I_{0}\left(k_{n} a\right) K_{1}\left(k_{n} a\right)}+\frac{q \kappa}{2 \epsilon_{w}} .
\end{aligned}
$$

In addition we will place two residues of charge $-q$ each embedded into the channel wall $(\rho=3 \AA)$ at positions $x=$ $-10.5 \AA$ and $x=10.5 \AA$, to represent the two binding sites of our transmembrane channel. The residues are placed inside the channel wall leaving space for the mobile ions to flow. The channel length and radius are comparable with the size of nanotubes and other models for the biological channels such as gA [10,35]. Although the gA channel does not have explicit charged residues, but only dipolar carbonyl groups [17,40], these behave similarly to the charged binding sites used in our model [10]. Furthermore, recent studies have shown that it is possible to use functionalized synthetic nanotubes to obtain ionic fluxes similar to the ones observed in the gA channel [35].

\section{B. Simulation details}

Ion channels can be highly selective to the cation passage, and nanotubes can be functionalized to mimic this behavior
$[1,8]$. Although the actual transport process is very complicated, we can identify three main steps [17]: (1) cation entry, where the positive ions are dehydrated, (2) cation translocation through the channel, and (3) cation exit. Since in our model we do not consider water molecules explicitly, we simulate step (1) above using a diameter for the cations equals to $\sigma_{+}=2 \AA$, while the negative ions have a diameter $\sigma_{-}=4 \AA$. Since the channel is very narrow, the available radius for ion movement is approximately $a-\sigma_{c} / 2$ in Fig. 1 . Therefore, using these diameters only positive ions can enter into the channel. In all interactions the energy parameter of $\mathrm{LJ}$ potential was defined as $\epsilon=1 k_{B} T$. During the MD steps, we have used Langevin dynamics to simulate the effect of solvent on the cation and anion movement, solving the equation of motion of ion $i$,

$$
m_{i} \vec{a}_{i}=\vec{F}_{i}-m \gamma \vec{v}_{i}+\vec{W}(t)
$$


where $\vec{F}_{i}$ is the total force on ion $i$ due to all entities explicitly present in the model (other ions, protein residues, and walls), $\gamma$ is the friction coefficient, and $\vec{W}(t)$ is the random force [41] due to solvent. The temperature of the system is maintained constant using the fluctuation-dissipation theorem,

$$
\left\langle\vec{W}(t) \cdot \vec{W}\left(t^{\prime}\right)\right\rangle=6 k_{B} T \gamma \delta\left(t-t^{\prime}\right),
$$

which relates the friction coefficient to the fluctuations of the random force using the appropriate $\gamma$ value. In our simulations we have used $m_{i}=6.5 \times 10^{-26} \mathrm{~kg}$, corresponding to the mass of $K^{+}$ion; $\gamma^{-1}=53$ fs for the region outside the channel, corresponding to diffusion constant $D=k_{B} T / m_{i} \gamma=3.37 \times$ $10^{-9} \mathrm{~m}^{2} / \mathrm{s}$; and $\gamma_{c}^{-1}=\gamma^{-1} / 3$ inside the channel corresponding to diffusion constant of $D=1.12 \times 10^{-9} \mathrm{~m}^{2} / \mathrm{s}$. These values were chosen in order to maintain the temperature fixed at $298 \mathrm{~K}$ and to reproduce the experimental behavior, particularly the saturation observed in the current-concentration curves [10].

We apply a linear voltage gradient across the membrane from the right border of $\mathrm{CV}_{1}$ to the left border of $\mathrm{CV}_{2}$. The concentrations in both reservoirs are maintained constant using DCV-GCMD simulations [30,31,42-46]. Briefly stated, in the DCV-GCMD simulation two control volumes (CVs) are initially prepared at desired concentrations using the grand canonical Monte Carlo (GCMC) simulation and then evolved in time using the molecular dynamics (MD) simulation. Since the dynamics alters the $\mathrm{CV}$ concentrations, the MD steps are intercalated with the GCMC simulations performed inside the two CVs shown in Fig. 1. This restores the concentrations to their initial values. In our simulations we have used 50 GCMC steps for every $500 \mathrm{MD}$ steps. It should be noted that our DCV-GCMD method is closely related to the GCMC/BD algorithm proposed by $\operatorname{Im}$ et al. [47] to simulate the ionic conductance in membrane channels.

Since our simulation setup is periodic only in the $y$ and $z$ directions, as shown in Fig. 1, for the long-range interactions described by Eq. (2) we have used the Ewald summation with the implementation of Yeh and Berkowitz [48] for the slab geometry. The equations of motion were integrated using the velocity Verlet algorithm, with a time step of $8.0 \mathrm{fs}$ in the MD simulations. The observables were obtained using 5 to 10 independent realizations.

\section{RESULTS AND DISCUSSION}

We start our discussion with the two CVs having the same concentration, and the ionic diffusion through the channel driven by the externally imposed electrostatic potential gradient between the two CVs. We are particularly interested in the current-voltage and current-concentration curves and their comparison with the experimental results, mainly the appearance of the experimentally observed saturation in the currentconcentration profiles. In Fig. 2 we show the current-voltage curve for the concentration of $0.5 \mathrm{M}$ in both CVs. As one can see, we obtain the same expected linear dependence between the ionic current and applied voltage, observed in experiments and earlier BD simulations for the gA channel [10].

Next we analyze the behavior of the current-concentration profiles for two externally applied voltages of 100 and $200 \mathrm{mV}$;

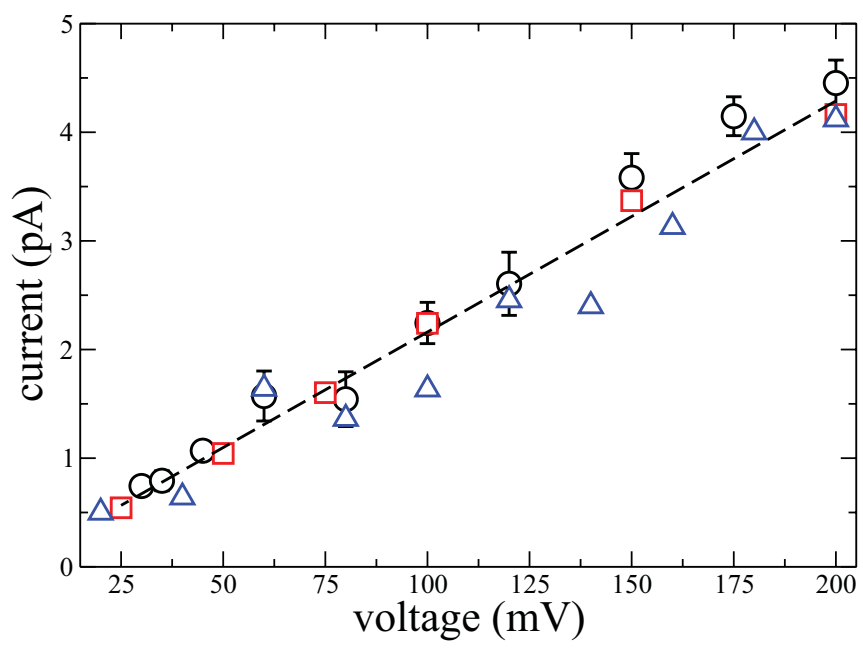

FIG. 2. (Color online) Current-voltage curve for $0.5 \mathrm{M}$ concentration of electrolyte in both CVs. Open circles are our simulations results. The open squares and the open triangle are the experimental and $\mathrm{BD}$ results for the $\mathrm{gA}$ channel, respectively, extracted from Fig. 12 of Ref. [10].

see Fig. 3. Compared to the experimental and BD simulation results [10], our model is able to capture the same saturation of the ionic current. For $200 \mathrm{mV}$ and above $0.9 \mathrm{M}$ concentration, however, we find some deviation from the experimental results. The deviation appears to be an artifact of including only 30 terms in the infinite series of the electrostatic potential inside the cell, Eqs. (5) and (6). Cutting the infinite series at only 30 terms softens the repulsion between the ions inside the channel favoring an increased flux.

Experimental [49-52] and BD simulations [10] data for the gA channel and theoretical results for functionalized nanotubes [35] have proposed two large concentration peaks at the binding sites separated by a cation-depleted region. This is exactly what we observe in our simulations, as shown in Fig. 4



FIG. 3. (Color online) Current-concentration curves for external voltages of 100 and $200 \mathrm{mV}$. Our simulations are represented by open circles. The open squares and open triangles are the experimental and BD results, respectively, extracted from Fig. 12 of Ref. [10]. 

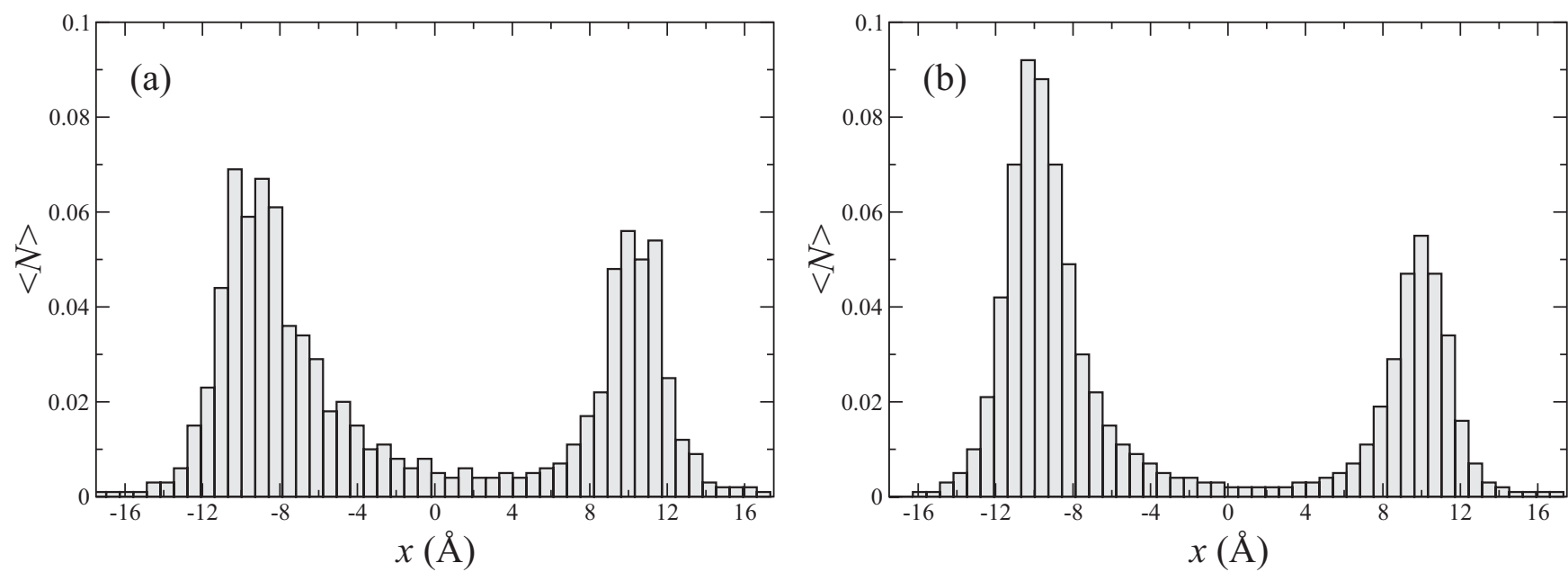

FIG. 4. Mean number of cations in the axial direction of the gA channel with (a) no applied voltage and (b) $200 \mathrm{mV}$ applied potential. In both cases there is $0.5 \mathrm{M}$ monovalent electrolyte solution in the two CVs.

for $0.5 \mathrm{M}$ monovalent solution in both CVs with no voltage and $200 \mathrm{mV}$ applied potential difference.

To understand better the mechanism of ionic translocation, in Fig. 5 we plot the electrostatic potential inside the transmembrane channel, Eq. (3), at zero applied voltage and for different occupancy inside the channel and residues embedded in the channel structure. These profiles were obtained using a dynamical PMF, where an ion, called ion*, is pulled through the channel, moving a displacement $\delta x$ each time. If there are other ions inside the channel (called ions 1, 2, 3, etc.) we move the ion*, and then we allow the system to relax for 4 ps.

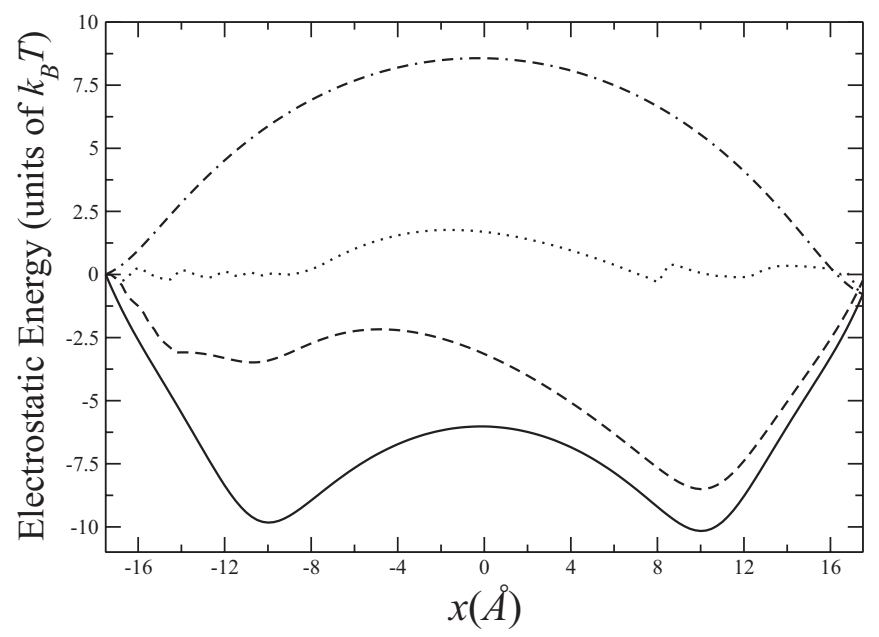

FIG. 5. Electrostatic energy profile inside the channel obtained from Levin's model [29]. The curves show the PMF felt by an ion moving through a gA-like channel with two charged residues at $x=-10.5 \AA$ and $x=+10.5 \AA$ (solid line). The PMF inside a channel that already contains a free cation near the first binding site is represented by a dashed line, while the PMF when there are two free cations near the two binding sites is represented by a doted line. The dot-dashed line represents the electrostatic potential produced by Eq. (7). The concentrations in the two CVs are $0.5 \mathrm{M}$, with no applied voltage.
During this relaxation time only ions 1,2 , etc., can move, while the ion* remains fixed. Then we evaluate the mean force on ion*, and perform the next displacement $\delta x$. The procedure is repeated until ion* leaves the channel. Note that during this process the other ions inside the channel will feel the electrostatic repulsion from ion* (recall that only cations are allowed inside the channel) and will also be forced out of the channel.

If the channel has no fixed residues and is empty, Eq. (7) leads to a large electrostatic potential energy barrier of approximately $8 k_{B} T$, which prevents any cation entrance. On the other hand, if the protein has charged residues embedded into the surface of the channel, the scenario changes completely. As one can see in solid line in Fig. 5, with no other cation inside the channel, the ion* feels an energy barrier of approximately $4 k_{B} T$ separating two deep wells at the positions of the binding sites.

Entrance of a cation alters drastically the potential energy landscape. Suppose that one ion, ion 1, is already inside the channel. We are interested in the PMF that a second ion, ion*, will feel as it moves through the channel. This PMF is plotted as a dashed curve in Fig. 5. A short time after the ion 1 has entered the channel its most probable location is at the first binding site. Thus, when ion* enters the channel, it sees the field of attractive residue partially screened by the ion 1 , so that the depth of the potential well produced by the first residue is significantly smaller. As the ion* moves farther into the channel, it forces the ion 1 to move to the position of the second residue and, eventually, to completely leave the channel. Consider now that there are two ions, ion 1 and ion 2 , inside the channel, with ion 1 located at the first binding site and ion 2 at the second binding site. The ion* will then encounter a flat energy landscape shown in Fig. 5 by the dotted curve. This then explains the fast transport of ions through the ion channel observed experimentally, the cations present at the binding sites screen the electric field produced both by the residues and by the induced charge on the channel wall. In the BD studies [10] the saturation was observed as the result of an imposed double-well potential of depth of $8 k_{B} T$ and a barrier between the wells of $5 k_{B} T$. In the present study, the 

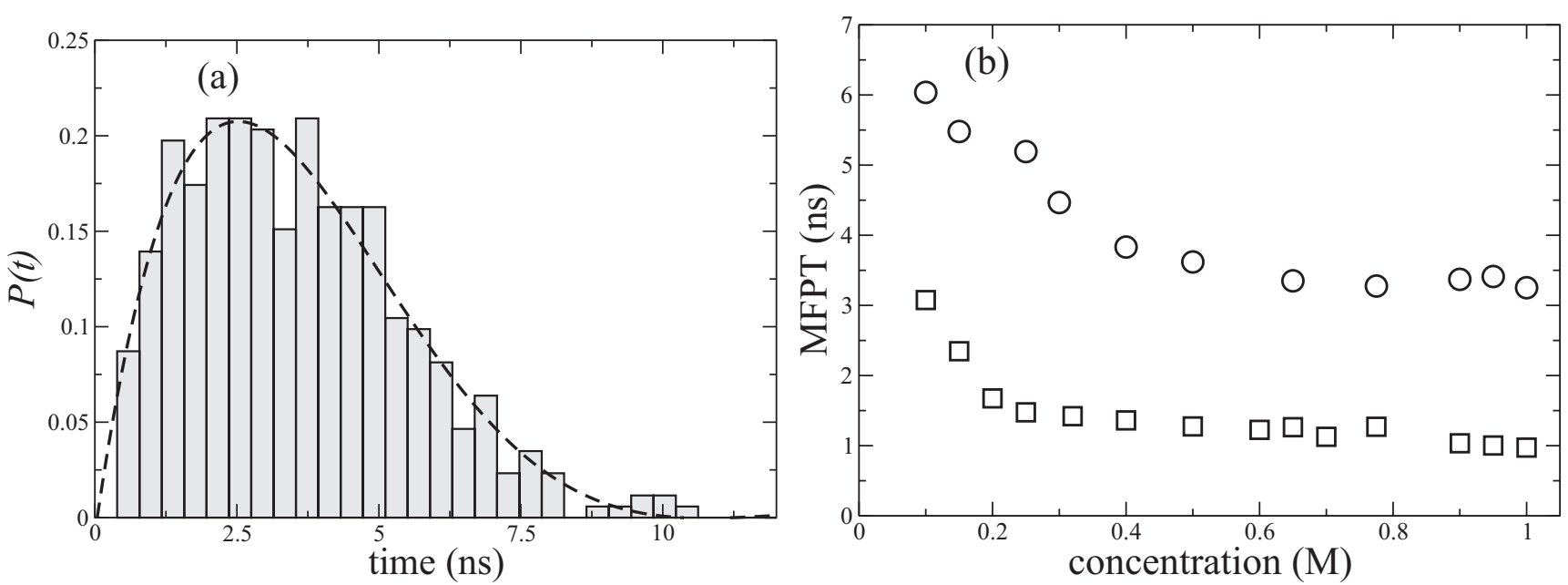

FIG. 6. (a) Distribution of passage time $P(t)$ for $100 \mathrm{mV}$ applied voltage and CV concentration of $0.5 \mathrm{M}$. (b) MFPT as a function of CV concentrations for $100 \mathrm{mV}$ (open circles) and $200 \mathrm{mV}$ (open squares) applied voltages.

electrostatic energy landscape was obtained self-consistently, assuming the existence of two monovalent binding sites inside the channel.

In Fig. 6 we show the distribution of times of cation translocation through the channel for voltage difference of $100 \mathrm{mV}$ and $\mathrm{CV}$ concentration of $0.5 \mathrm{M}$. The mean-firstpassage time (MFPT) as a function of applied voltage and CVs concentration is also shown in Fig. 6. The saturation observed in Fig. 3 can also be seen in the MFPT results.

\section{CONCLUSIONS}

We have used Dual-Control-Volume Grand-Canonical Molecular Dynamics (DCV-GCMD) simulations to study the flow of ions through narrow pores across low dielectric membranes. To account for the electrostatic interactions inside the channel we have used the analytical potential recently derived by Levin [29]. For electrolyte concentrations up to $1 \mathrm{M}$ our model is able to reproduce most of experimental behavior of the ionic current as a function of electrostatic potential difference. To obtain reliable results for larger concentrations one must include more terms in the infinite series for the ion-ion interaction potential. For physiological concentrations of electrolytes, on the other hand, the continuum model presented in this work appears to show excellent results. The utility of the model is that the simulations do not require expensive computational resources and can be run on a desktop PC. One interesting application of the model is to study the dependence of ionic current on mutations of charged residues: The position and the charge of the residues that enter into the electrostatic potential can be optimized to obtain the desirable channel characteristics. This could be of interest for design and implementation of molecular engineering of ion channels and nanopores, biological or synthetic.

\section{ACKNOWLEDGMENTS}

This work was partially supported by the CNPq, Capes, Fapergs, and INCT-FCx and by the US-AFOSR under grant FA9550-09-1-0283.
[1] B. Hille, Ion Channels of Excitable Membranes, 3rd ed. (Sunderland, MA: Sinauer Associates, 2001).

[2] D. A. Doyle, J. M. Cabral, R. A. Pfuetzner, A. Kuo, J. M. Gulbis, S. L. Cohen, B. T. Chait, and R. MacKinnon, Science 280, 69 (1998).

[3] S. Berneche and B. Roux, Nature (London) 414, 73 (2001).

[4] P. J. F. Harris, Carbon Nanotubes and Related Structures: New Materials of the Twenty-First Century (Cambridge University Press, Cambridge, 1999).

[5] X. Shi, Y. Kong, and H. Gao, Acta Mech. Sin. 24, 161 (2008).

[6] T. A. Hilder, D. Gordon, and S. H. Chung, Biophys. J. 99, 1734 (2010).

[7] T. A. Beu, J. Chem. Phys. 132, 164513 (2010).

[8] T. A. Hilder, D. Gordon, and S. H. Chung, Nanomedicine 7, 702 (2011).
[9] B. Roux, Biophys. J. 71, 3177 (1996).

[10] S. Edwards, B. Corry, S. Kuyucak, and S. Ho Chung, Biophys. J. 83, 1348 (2002).

[11] B. Roux, T. W. Allen, T. W. Bernèche, and W. Im, Q. Rev. Biophys. 37, 15 (2004).

[12] S. Joseph, R. J. Mashl, E. Jakobsson, and N. R. Aluru, Nano Lett. 3, 1399 (2003).

[13] M. Majumder, X. Zhan, R. Andrews, and B. J. Hinds, Langmuir 23, 8624 (2007).

[14] T. A. Hilder, D. Gordon, and S. H. Chung, J. Chem. Phys. 134, 045103 (2011).

[15] D. G. Levitt, J. Gen. Physiol. 113, 789 (1999).

[16] B. Roux, Curr. Opin. Struct. Biol. 12, 182 (2002).

[17] O. S. Andersen, R. E. Koeppe, and B. Roux, IEEE Trans. Nanobiosci. 4, 10 (2005).

[18] A. Aksimentiev and K. Schulten, Biophys. J. 88, 3745 (2005). 
[19] D. E. Shaw, P. Maragakis, K. Lindorff-Larsen, S. Piana, R. O Dror, M. P. Eastwood, J. A. Bank, J. M. Jumper, J. K. Salmon, Y. Shan, and W. Wriggers, Science 330, 341 (2010).

[20] T. W. Allen, O. S. Andersen, and B. Roux, Biophys. J. 90, 3447 (2006).

[21] T. W. Allen, O. S. Andersen, and B. Roux, Biophys. Chem. 124, 251 (2006).

[22] H. I. Ingólfsson, Y. Li, V. V. Vostrikov, H. Gu, J. F. Hinton, R. E. Koeppe II, B. Roux, and O. S. Andersen, J. Phys. Chem. B 115, 7417 (2011).

[23] M. D. Baer and C. J. Mundy, J. Phys. Chem. Lett. 2, 1088 (2011)

[24] Y. Levin, A. P. dos Santos, and A. Diehl, Phys. Rev. Lett. 103, 257802 (2009).

[25] A. P. dos Santos, A. Diehl, and Y. Levin, Langmuir 26, 10778 (2010).

[26] G. Moy, B. Corry, S. Kuyucak, and S. H. Chung, Biophys. J. 78, 2349 (2000).

[27] B. Corry, S. Kuyucak, and S. H. Chung, Biophys. J. 78, 2364 (2000).

[28] R. Carr, J. Comer, M. D. Ginsberg, and A. Aksimentiev, Lab Chip 11, 3766 (2011).

[29] Y. Levin, Europhys. Lett. 76, 163 (2006).

[30] G. S. Heffelfinger, and F. Van Smol, J. Chem. Phys. 100, 7548 (1994).

[31] A. P. Thompson, D. M. Ford, and G. S. Heffelfinger, J. Chem. Phys. 109, 6406 (1998).

[32] R. S. Eisenberg, J. Membr. Biol. 171, 1 (1999).

[33] D. G. Luchinsky, R. Tindjong, I. Kaufman, P. V. E. McClintock, and R. S. Eisenberg, Phys. Rev. E 80, 021925 (2009).
[34] Y. Levin, Rep. Prog. Phys. 65, 1577 (2002).

[35] T. A. Hilder and S. H. Chung, Chem. Phys. Lett. 501, 423 (2011).

[36] Y. Li, O. S. Andersen, and B. Roux, J. Phys. Chem. B 114, 13881 (2010).

[37] T. W. Allen, O. S. Andersen, and B. Roux, J. Gen. Physiol. 124, 679 (2004).

[38] M. G. Kurnikova, R. D. Coalson, P. Graf, and A. Nitzan, Biophys. J. 76, 642 (1999).

[39] M. P. Allen and D. J. Tildesley, Computer Simulation of Liquids (Oxford University Press, Oxford, 1987).

[40] R. R. Ketchem, B. Roux, and T. A. Cross, Structure 5, 1655 (1997).

[41] N. Wiener, J. Math. Phys. 2, 131 (1923).

[42] A. V. Raghunathan and N. R. Aluru, Phys. Rev. E 76, 011202 (2007).

[43] G. S. Heffelfinger and D. M. Ford, Mol. Phys. 94, 659 (1998).

[44] D. M. Ford and G. S. Heffelfinger, Mol. Phys. 94, 673 (1998).

[45] P. I. Pohl and G. S. Heffelfinger, J. Membr. Sci. 155, 1 (1999).

[46] M. Horsch and J. Vrabec, J. Chem. Phys. 131, 184104 (2009).

[47] W. Im, S. Seefeld, and B. Roux, Biophys. J. 79, 788 (2000).

[48] M. L. Berkowitz and I. Yeh, J. Chem. Phys. 111, 3155 (1999).

[49] F. Tian and T. A. Cross, J. Mol. Biol. 285, 1993 (1999).

[50] D. W. Urry, T. L. Trapane, C. M. Venkatachalam, and R. B. McMichens, Methods Enzymol. 171, 286 (1989).

[51] G. A. Olah, H. W. Huang, W. Liu, and Y. Wu, J. Mol. Biol. 218 , 847 (1991).

[52] L. V. Schagina, A. E. Grinfeldt, and A. A. Lev, Nature (London) 273, 243 (1978). 